\title{
The preceding item as cue for a response in serial learning'
}

BENJAMIN MCKEEVER, DEPARTMENT OF PSYCHOLOGY UNIVERSITY OF WASHINGTON, Seattle, Wash. 98105

This experiment was a further test of the hypothesis that in serial learning an item serves as an effective stimulus for the next item. All Ss studied the same four lists, each of eight words. Half then learned a list of four pairs of words, the pairs selected from different original lists but each consisting of previously adjacent words. The other Ss also learned a list of eight words selected from the origina! items, but all previously nonadjacent. For both groups each word had the same serial position in both its original and derived list. The findings are interpreted as consistent with the hypothesis tested.

Recent research on the nature of the functional stimulus in serial learning (SL) (much of it reviewed by Jensen \& Rohwer, 1965) has provided impressive evidence of the effectiveness of serial position as a cue for a response (e.g., Ebenholtz, 1963a, b; Slamecka, 1967). But there is also recently obtained experimental support for the traditional "chaining hypothesis," according to which sequential associations are formed between an item and one or more of its predecessors (e.g., Battig, Brown, \& Schild, 1964; Postman \& Stark, 1967; Shuell \& Keppel, 1967).

The aim of the present experiment was to verify further the acquisition of such associations. Specifically, its purpose was another test of the hypothesis that in SL a single item serves as an effective stimulus for the adjacently succeeding item. Using an SL to SL transfer design, the experiment required the learning of several original lists, with degree of adjacency of their items later varied for two groups of Ss on transfer lists. In addition, the effect of positional cues was controlled by assigning each item to the same serial position in both the original and the transfer learning.

Lists. All Ss, run individually, studied successively the same four lists of eight words each. Each $S$ then learned a fifth list of the same length, selected from one of two sets of four lists each, all derived from the original lists.

One set of derived lists (DL) was constructed as follows. To obtain the first list of the set, an item randomly selected from the four items in the first serial position of the original lists was made the first item on the new list. Next, the second item was randomly chosen from the three items in the same position in the lists not previously sampled. Similarly, the third and fourth items were selected from the third and fourth items, respectively, of the lists as yet unsampled. The fifth through eighth items were taken, in order, from each of the lists that had provided items one through four. Three additional DLs of this set were constructed similarly, but with the restriction that all the items selected for a given list should not have been used on any previously constructed DL of the set. Thus, the four lists obtained (the No-AI lists) had these characteristics: (1) they consisted of all the 32 items of the original lists; (2) each item had the same serial position in both its original list and DL; (3) no items adjacent on a DL had fewer than three degrees of remoteness from one another in any original list.

The second set of four DL (the AI lists) also consisted of all the items of the original lists. Each list of this set, however, was derived by selecting four pairs of adjacent items from the original lists in a manner satisfying the following conditions: (1) each pair consisted of an item in an odd-numbered serial position and the item adjacently succeeding it; $(2)$ as in the case of the No-AI lists, all items retained their original serial positions in the new lists; (3) the pairs of a given list were randomly selected, with the restrictions that they should be taken from different original lists and each should be assigned to only one list of the set.

The list items were 32 words selected from the Minnesota norms of Palermo \& Jenkins (1964) and were without interitem associations according to these norms.

Procedure. The Ss, 48 females fulfilling a course requirement in introductory psychology by their participation, were randomly assigned to one of two equal groups, the Al group and the No-AI group. Each original list was presented by a memory drum for 10 trials, the order of list presentation for each $\mathrm{S}$ of a given group being a different one of the 24 permutations of the lists. Learning was by the anticipation method with a 2-sec exposure of each item. Approximately $15 \mathrm{sec}$ elapsed between presentations of the different lists and $4 \mathrm{sec}$ between trials. An asterisk signalled the appearance of the first item.

Approximately $30 \mathrm{sec}$ after original learning (OL), equal numbers of AI and No-AI Ss were randomly assigned the task of learning one of the four AI lists or one of the four No-Al lists, respectively. The instructions stated that the words in these lists had appeared in the previous lists, but made no reference to their serial positions. The conditions of presentation for the DL were the same as in OL, except that the DL were learned to a criterion of one errorless trial

Results. The mean number of errors made by the AI and No-AI Ss on the original lists, 42.71 and 47.50 , respectively, were not significantly different $(t=.74$, df $=46)$. Nor was the mean number of trials required by the Al group to reach criterion on the DL, 2.58, reliably different from that of the No-AI Ss, $3.17(t=$ 1.34 , df $=46$ ). However, the groups did show significant differences in the frequency of their errors on the even-numbered words of their respective DL, i.e., the words which in the AI lists, but not in the No-AI lists, adjacently succeeded the same items as in OL. The total number of AI errors on these words was less than that of No-AI errors (Table 1), a difference found significant by the Mann-Whitney $U$ test $(U=387.5, z=2.09, p<.05)$. (This test, with correction for ties, was used in view of the extreme skewness of the error distributions obtained. All $p$ values reported are for two-tailed hypotheses.) On the first trial alone, the AI errors on the even-numbered words were also fewer than the corresponding No-Al errors, a difference closely approaching the conventional level of signifjcance $(U=376, z=1.88, p<.07)$. The groups differed most strikingly, however, in the frequency of a particular class of errors on the even-numbered words of their respective DL. These were errors following correct anticipations of the adjacently preceding items, i.e., the odd-numbered words of the lists. AI errors of this class were significantly fewer than the No-AI errors, both on the first trial of the DL learning $(U=394.5$, $\mathrm{z}=2.49, \mathrm{p}<.02)$ and on all trials $(\mathrm{U}=497, \mathrm{z}=2.58, \mathrm{p}<.01)$.

In contrast to their performance on the even-numbered words, the groups did not differ significantly in number of errors on the odd-numbered items of their DL, i.e., words not adjacent for either group to the same items as in $\mathrm{OL}(\mathrm{U}=341, \mathrm{z}=1.12)$. Furthermore, the frequency of errors on these words following correct anticipations of the immediately preceding items was nearly identical for the two groups, both on the first trial of the $\mathrm{DL}$ learning $(\mathrm{AI}=16 ; \mathrm{No}-\mathrm{AI}=15)$ and on all trials $(\mathrm{AI}=31$; $\mathrm{No}-\mathrm{AI}=30$ ).

Discussion These findings seem to support two inferences: (1) sequential associations between adjacent items were acquired in $\mathrm{OL} ;(2)$ consequently, the odd-numbered words of the $\mathrm{AI}$ lists were effective cues to the immediately succeeding even-numbered items, i.e., words also adjacent to the former in the original lists. The formation of sequential associations in $\mathrm{OL}$, and their "use" in acquiring the Al lists, is implied by the findings of significantly fewer errors on these even-numbered AI words than on the corresponding No-Al words, but of no significant difference between the groups in the frequency of errors on the odd-numbered items of their respective DL, i.e., words which for both groups followed different items

Table 1

Means of Errors on Odd and Even Words of the $D L^{(a)}$

\begin{tabular}{lccccc} 
& \multicolumn{2}{c}{ Trial 1 } & & \multicolumn{2}{c}{ All Trials } \\
\cline { 2 - 3 } \cline { 5 - 6 } Words & AI & No-AI & & Al & No-Al \\
\hline Odd & $1.29(.67)$ & $1.45(.62)$ & $2.04(1.29)$ & $2.75(1.25)$ \\
Even & $1.00(.29)$ & $1.62(.75)$ & $1.58(.71)$ & $3.08(1.67)$ \\
\hline
\end{tabular}

(a) Means of errors following correct anticipations in parentheses. 
from those in OL. Further, and stronger, evidence for this conclusion is the difference between the groups in the frequency of errors on their respective even-numbered words following correct anticipations of the immediately preceding items. When such anticipations were made, it is evident that if sequential associations had been acquired in OL, not only seeing, but saying, the odd-numbered words could serve the AI Ss as cues for the adjacently succeeding items. Apparently this in fact occurred. For, as already noted, following the correct anticipations in question the frequency of errors on the even-numbered words of the AI lists was markedly less than that of the corresponding No-AI errors, a difference significant beyond the $1 \%$ level.

Thus, two general conclusions are suggested. (1) Since the AI pairs of words adjacent in OL were chosen from different original lists, the findings seem consistent with the hypothesis tested, namely, that in SL a single item is an effective stimulus for the next item. (2) Furthermore, apparently either stronger sequential than positional associations were acquired in $O L$, or else the former showed more transfer than the latter to the DL learning. This would seem evident from two considerations. (a) Since each item in both the $\mathrm{AI}$ and No-Al lists retained the same serial position it had in $\mathrm{OL}$, both the $\mathrm{AI}$ and No-Al group presumably benefited equally in the DL learning from any transfer due to positional learning in OL. (b) Yet, when the AI Ss were apparently also aided in the DL learning by sequential associations acquired in $O L$. this group made significantly fewer errors than did the No-Al group.

\section{REFERENCES}

BATTIG, W. F., BROWN, S. C., \& SCHILD, M. E. Serial position and sequential associations in serial learning. J. exp. Psychol, 1964, 67, 449-57.

EBENHOLTZ, S. M. Serial learning: Position learning and sequential associations. J. exp. Psychol., 1963a, 66, 353-362.

EBENHOLTZ, S. M. Position mediated transfer between serial learning and a spatial discrimination task. J. exp. Psychol., 1963b, 65, 603-608.

JENSEN, A. R., \& ROHWER, W. D., Jr. What is learned in serial learning? $J$. verbal Learn. verbal Behav., 1965, 4, 62-67.

PALERMO, D. S., \& JENKINS, J. Word association norms: Grade School through College. Minneapolis: Univ. Minnesota Press, 1964.

POSTMAN, L. \& STARK, K. Studies of learning to learn: IV. Transfer from serial to paired associate learning. J. verbal Learn. verbal Behav., 1967, 6, 339-353.

SHUELL, T. J., \& KEPPEL, G. A further test of the chaining hypothesis of serial learning. J. verbal l,earn, verbal Behav., 1967, 6, 439-445.

SLAMECKA, N. Serial learning and order information. J. exp. Psychol., $1967,74,62-66$

\section{NOTE}

1. Supported by the Graduate Research Fund of the University of Washington. Appreciation is expressed to Mr. James Skucy for his assistance in gathering the data. 